\title{
Enhanced Group Delay of the Pulse Reflection with Graphene Surface Plasmon via Modified Otto Configuration
}

\author{
Guimei Li, ${ }^{1}$ Qiwei Liu, ${ }^{2}$ Yue Liu, ${ }^{2}$ Yueying Wei, ${ }^{2}$ Rui Weng, \\ Yuxiang Zhou, ${ }^{2}$ and Leyong Jiang ${ }^{2}$ \\ ${ }^{1}$ School of Computer and Information Engineering, Hunan University of Commerce, Changsha 410205, China \\ ${ }^{2}$ College of Physics and Information Science, Hunan Normal University, Changsha 410081, China \\ Correspondence should be addressed to Leyong Jiang; jiangly28@hunnu.edu.cn
}

Received 28 June 2017; Accepted 30 August 2017; Published 1 November 2017

Academic Editor: Yan Luo

Copyright (C) 2017 Guimei Li et al. This is an open access article distributed under the Creative Commons Attribution License, which permits unrestricted use, distribution, and reproduction in any medium, provided the original work is properly cited.

In this paper, the group delay of the transverse magnetic (TM) polarized wave reflected from a modified Otto configuration with graphene surface plasmon is investigated theoretically. The findings show that the optical group delay in this structure can be enhanced negatively and can be switched from negative to positive due to the excitation of surface plasmon by graphene. It is clear that the negative group delay can be actively tuned through the Fermi energy of the graphene. Furthermore, the delay properties can also be manipulated by changing either the relaxation time of graphene or the distance between the coupling prism and the graphene. These tunable delay characteristics are promising for fabricating grapheme-based optical delay devices and other applications in the terahertz regime.

\section{Introduction}

The group delay properties of optical pulse traversing through a system together with delay control techniques have received extensive research interests due to their importance in optical communication $[1,2]$. The pulse delay control techniques enable unique micro/nanodevices with novel functionalities. The potential applications of the above group delay properties include controllable all-optical delays [3], arbitraryangle beamforming [4], and all-optical buffers [5]. Various techniques and approaches have been explored to accomplish the tunable and large group delay in order to achieve the extraordinary phenomena described as "subluminal effect" and "superluminal effect" in various frequency ranges, like electromagnetically induced transparency (EIT) systems [6], weakly absorbing dielectric [7], Fabry-Perot cavity configuration [8], metamaterial slab [9], and so on. Compared with the subluminality, the superluminality characterized by negative group delay continues to attract much attention due to the counterintuitive phenomenon and the possible applications. For example, Manipatruni et al. realized superluminal propagation on a silicon microchip by using coherent interaction between the microcavities [10]; Choi et al. reported an enhancement of feedforward amplifiers by adopting superluminal delay circuit [11]. More recently, Yao et al. experimentally demonstrated a negative group delay approach in a circular waveguide system with an asymmetric cross-shaped slotted configuration [12]. However, the demands to develop optical delay approach with large delay time, high tenability, and easy integration for optical communication are ever increasing.

In recent years, graphene, a two-dimensional atomic crystal material, has received a wide range of attention in the field of optoelectronics due to its excellent optical and electrical properties, such as zero-gap characteristic [13], electrical tunability $[14,15]$, low losses $[16,17]$, and strong nonlinear response [18], among others. It is worth mentioning that graphene can support the existence of TM polarized and transverse electric (TE) polarized surface plasmon polaritons (SPPs) propagating along graphene, depending on the sign of the imaginary part of graphene conductivity [19]. This feature can significantly enhance the light-matter interaction [20] and further enriches its application in the terahertz $(\mathrm{THz})$ and infrared spectrum [21-23]. It is known that the large 


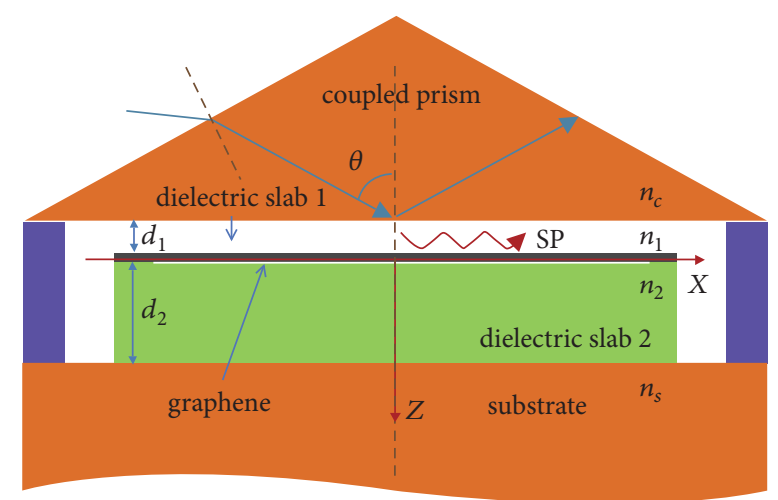

FIGURE 1: Schematic diagram of a modified Otto configuration with the insertion of monolayer graphene. An incident light with an angle $\theta$ to the surface normal is considered.

group delay is usually found near a resonance of the structure. Hence, the combination of graphene and surface plasmon obviously seems to be an alternative candidate for realizing and manipulating tunable group delay devices.

In this paper, it is proposed and theoretically demonstrated that there is a possibility of realizing and manipulating large reflected group delay of optical pulse from a modified Otto configuration with the graphene surface plasmon in $\mathrm{THz}$ regime. This large reflected group delay originates from the local abrupt phase change at the interface of two dielectrics with monolayer graphene owing to the excitation of the surface plasmon. In particular, since the optical conductivity of graphene can be dynamically manipulated by varying the Fermi energy, the corresponding subluminal and superluminal effects can be continuously tuned in a frequency regime. The pathway proposed in this paper is designed to manipulate the reflected group delay of $\mathrm{THz}$ pulses, which would inspire the potential for a variety of applications [24-26], particularly for sensing technologies and $\mathrm{THz}$ delay devices.

\section{Theoretical Model and Method}

In this section, an attenuated total reflection scheme for exciting SPPs is under consideration using modified Otto configuration [27], in which a dielectric layer refractive index with thickness $d_{2}$ is placed on a substrate. Dielectric slab 2 is covered by monolayer graphene. Between dielectric slab 2 and the coupled prism there is the dielectric slab 1 with a thickness of $d_{1}$ as shown in Figure 1, where $z$ direction is the incidence direction, while $x$ direction is parallel to the plane of the monolayer graphene. $n_{1}$ and $n_{2}$ are the refractive index of dielectric slab 1 and the refractive index of dielectric slab 2 , respectively. $n_{c}$ and $n_{s}$ are the refractive index of coupled prism and the refractive index of substrate. Here, to facilitate the excitation of SPPs, dielectric slab 1 is assumed to be an air layer with low refractive index and small thickness. In the following calculations, the center frequency is $5 \mathrm{THz}$, temperature $T=300 \mathrm{~K}$, and relaxation time $\tau=1 \mathrm{ps}$. Besides, in order to inhibit the absorption of incident electromagnetic waves caused by the excitation of SPPs, the thicknesses of dielectric slab 1 and dielectric slab 2 in the configuration are set as $2 \mu \mathrm{m}$ and $8 \mu \mathrm{m}$, respectively. Meanwhile, to facilitate the excitation of SPPs, dielectric slabs 1 and 2 are assumed to be low refractive air and polymethylpentene material, whose respective indexes are $n_{1}=1$ and $n_{2}=1.46$, respectively. In addition, high refractive materials in $\mathrm{THz}$ spectrum are used for the coupling prism and substrate. More specifically, germanium materials (refractive index $n_{\mathrm{c}}=n_{s}=3.84$ ) are selected for the prism and substrate in this research. For the sake of simplicity, the dielectric loss is not taken into consideration, which means the imaginary part of refractive index is assumed to be zero. The optical properties of the monolayer graphene can be represented by optical conductivity. Under random phase approximation, the surface conductivity of graphene can be regarded as the sum of interconductivity and intraconductivity [28]. However, the surface conductivity of graphene in the terahertz frequencies is dominated by the intraconductivity. At this moment, the graphene conductivity can be approximately expressed as

$$
\sigma=\frac{i e^{2} k_{B} T}{\pi \hbar^{2}(\omega+i / \tau)}\left(\frac{E_{F}}{k_{B} T}+2 \ln \left(e^{-E_{F} / k_{B} T}+1\right)\right),
$$

where $e, K_{B}, T$, and $\hbar$ represent the electron charge, Boltzmann constant, temperature, and reduced Planck's constant, respectively; $\omega$ represents the angular frequency of incident electromagnetic wave; $\tau$ and $E_{F}$ represent the electronphonon relaxation time and the Fermi energy of graphene, respectively. The above equation shows that the conductivity properties of graphene are closely related to Fermi energy, and the value of Fermi energy can be tuned by external voltage, which provides a means to flexibly tune the optical conductivity of graphene and further tune the optical properties of the whole configuration.

In order to calculate the reflected group delay properties of the configuration, the transmission and reflection properties of the whole configuration are also required. Based on the monoatomic layer characteristic of the graphene, the modified transfer matrix method is adopted in this paper to calculate the transmittance and reflectance of the configuration [29]. In this paper, as graphene is just of monoatomic thickness, its conductivity properties can be reflected in boundary conditions. For this reason, the transmission matrix between the TM polarized dielectric slab 1 and dielectric slab 2 can be expressed as

$$
D_{12}=\frac{1}{2}\left[\begin{array}{ll}
1+\eta_{\mathrm{TM}}+\xi_{\mathrm{TM}} & 1-\eta_{\mathrm{TM}}-\xi_{\mathrm{TM}} \\
1-\eta_{\mathrm{TM}}+\xi_{\mathrm{TM}} & 1+\eta_{\mathrm{TM}}-\xi_{\mathrm{TM}}
\end{array}\right] .
$$

In this equation, $\eta_{\mathrm{TM}}=\varepsilon_{1} k_{2 z} / \varepsilon_{2} k_{1 z}, \xi_{\mathrm{TM}}=\sigma k_{2 z} / \varepsilon_{0} \varepsilon_{2} \omega$, where $k_{1 z}$ and $k_{2 z}$ represent the components of wave vectors $k_{1}$ and $k_{2}$ in the transmission direction of electromagnetic wave; $\varepsilon_{0}$, $\varepsilon_{1}$, and $\varepsilon_{2}$ are the vacuum permittivity, dielectric constants of dielectric slab 1, and dielectric constants of dielectric slab 2, respectively. Similarly, the TE polarized transmission matrix is also presented in [29]. However, it is noteworthy that graphene can excite both TE polarized and TM polarized SPPs, which is quite different from metal as the latter can only excite TM polarized SPPs. But the two polarized 


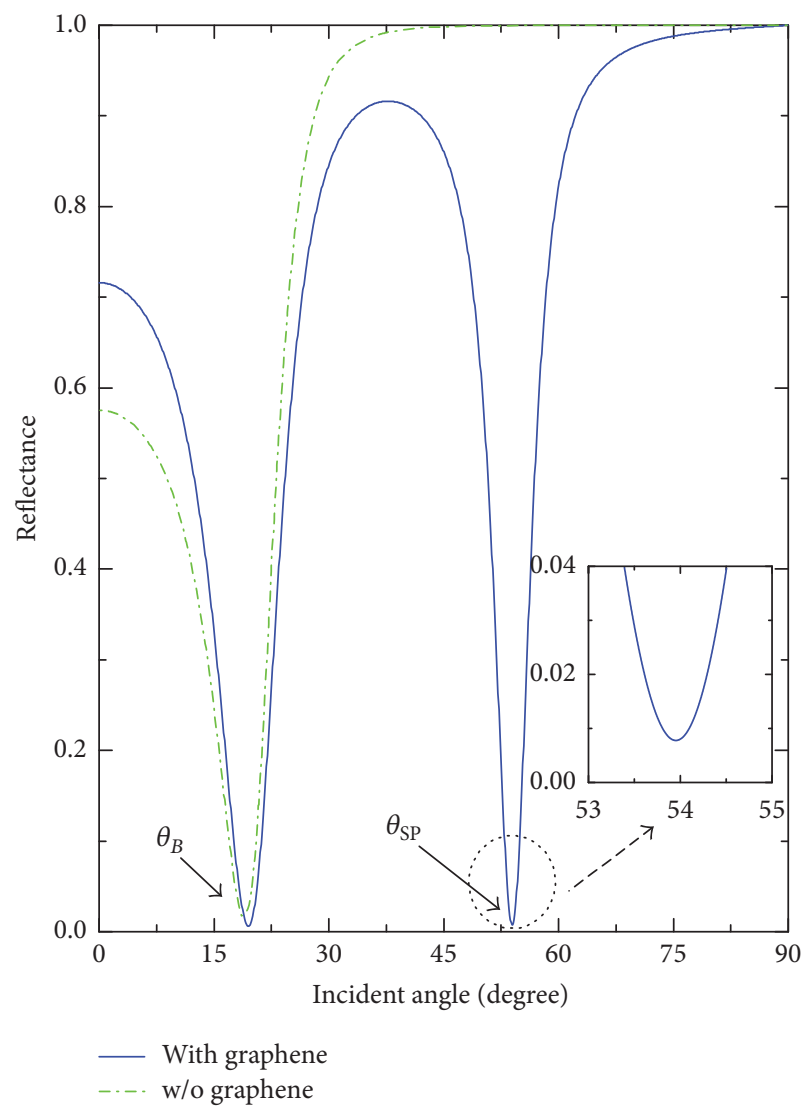

(a)

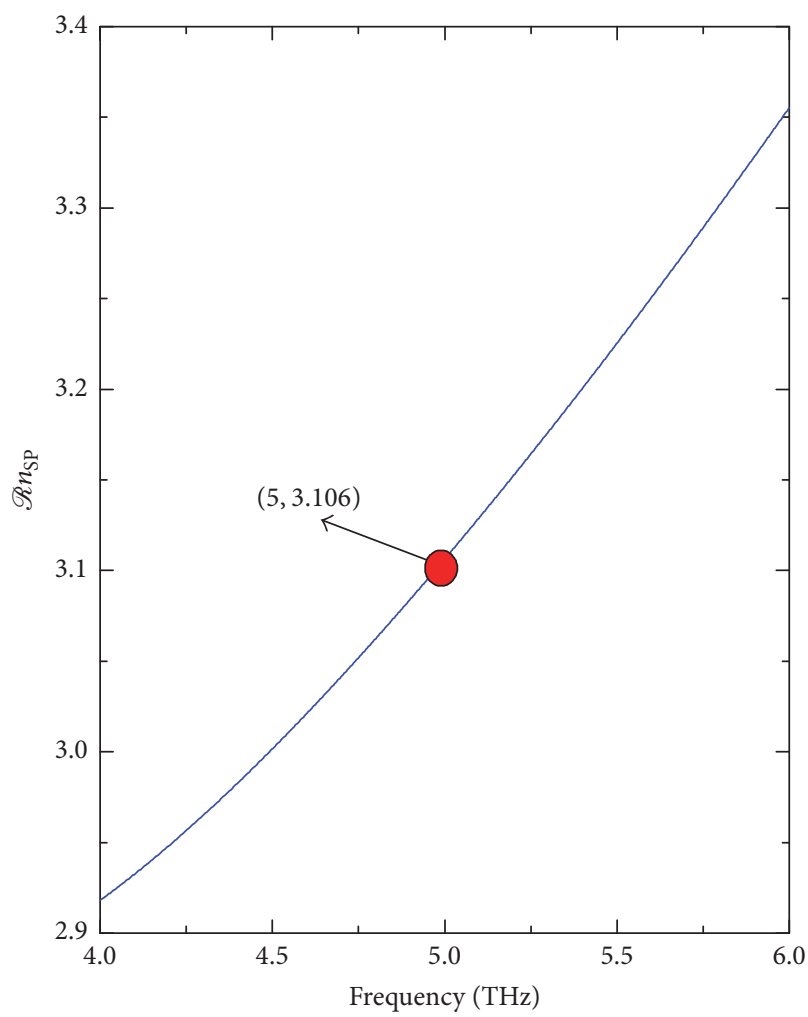

(b)

FIGURE 2: (a) Reflectance of the configuration as a function of incident angle $\theta$ (solid line). For comparison, the reflectance without monolayer graphene is also shown (dash dot line). (b) SPPs dispersion characteristics on monolayer graphene as a function of frequency.

exciting spectrums should not be in the same spectrum [19], and this research only considers the TM polarized pulse reflected group delay. Based on the transmission matrix and the propagation matrix transmitted in the dielectric layer, the transmission coefficient and reflection coefficient of the whole configuration can be obtained. If the incident pulse is assumed to be Gaussian pulse, the reflected group delay of the whole configuration can be expressed as

$$
\tau_{r}=\left[\frac{\partial \phi_{r}}{\partial \omega}\right]_{\omega=\omega_{c}} .
$$

Here, $\omega_{c}$ is the carrier frequency, $\phi_{r}$ is the phase of the reflection coefficient $r(\omega)$, and $r(\omega)=|r(\omega)| \exp \left(i \phi_{r}(\omega)\right)$.

\section{Results and Discussions}

3.1. Reflectance and Dispersion Relation. In this section, the characteristics of reflected group delay in the configuration will be discussed. In respect to the lossless configuration and dielectric, the zero reflection at the resonances $(\operatorname{Re}(k d)=$ $m \pi(m=1,2,3, \ldots))$ would make the reflected delay at the resonances physically meaningless. However, when monolayer graphene is embedded into the contact surface of the dielectric layer with excited SPPs, the reflectance will become very small but not turn to zero, and the slope of the reflected phase will be changed as well. Consequently, the conditions for realizing large reflected group delay are created. Besides, the tunable optical characteristics give graphene considerable advantages in terms of controllable enhanced pulse delay. In the following calculation, it is assumed that the Fermi energy satisfies $E_{F}=1 \mathrm{eV}$. First, the reflection characteristics of the whole configuration are illustrated in Figure 2(a). When monolayer graphene is missing, it is impossible to excite SPPs in the configuration. As a result, there is only one reflectance dip at the Brewster angle $\left(\theta_{B}\right)$ among the reflectance that changes with the angle. But when the incident angle is larger than Brewster angle, the reflectance of the whole configuration is almost $100 \%$ due to total reflection. Meanwhile, as the dielectric losses are neglected, the transmittance is almost zero (not shown in the figure). However, when the graphene is embedded into the configuration and excites SPPs through proper wave vector matching, a second reflectance dip occurs near the $54^{\circ}$ larger than the Brewster angle. As shown in Figure 2(a), the dip is narrow and the reflectance approximates to zero. To describe the generation mechanism of the dip, an SPPs dispersion characteristics curve of the configuration is drawn based on boundary conditions, as shown in Figure 2(b). According to this curve, in the configuration proposed in this paper, the effective 

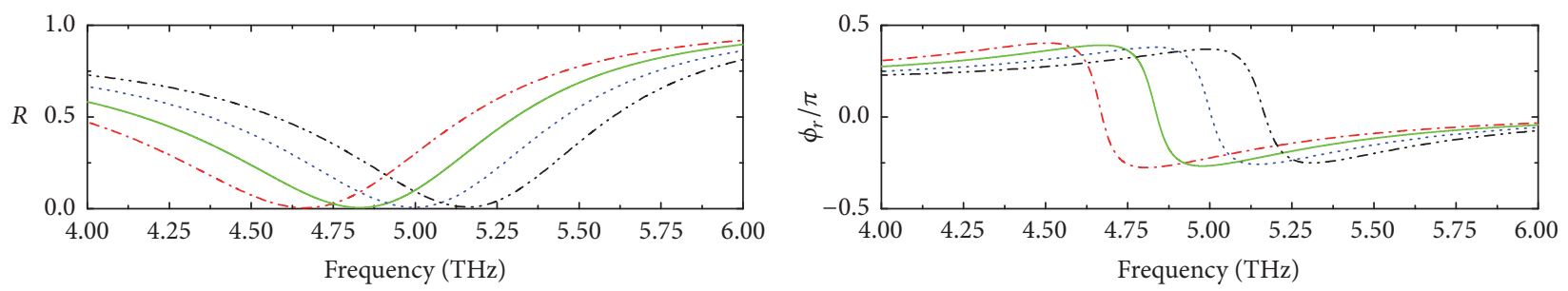

$$
\begin{aligned}
& \text { ‥ } E_{F}=0.96 \mathrm{eV} \\
& -E_{F}=0.98 \mathrm{eV} \\
& \text { … } E_{F}=1.00 \mathrm{eV} \\
& -\cdots-E_{F}=1.02 \mathrm{eV}
\end{aligned}
$$

(a)

$$
\begin{aligned}
\ldots E_{F} & =0.96 \mathrm{eV} & \cdots & E_{F}=1.00 \mathrm{eV} \\
-E_{F} & =0.98 \mathrm{eV} & -\cdots- & E_{F}=1.02 \mathrm{eV}
\end{aligned}
$$

(b)

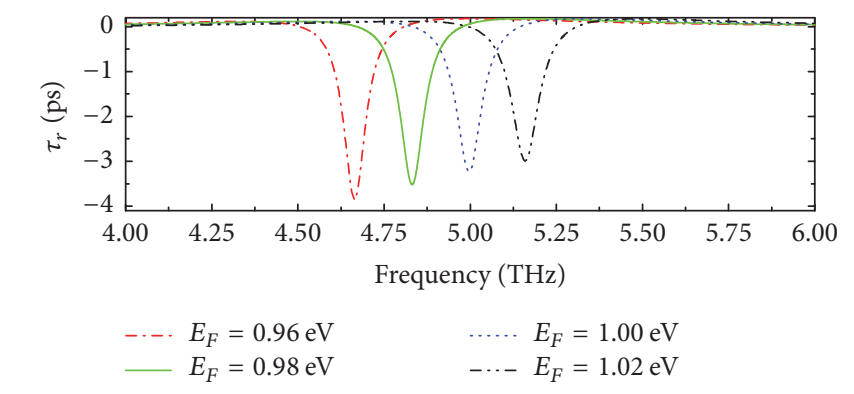

(c)

Figure 3: (a) Reflectance $R$, (b) reflected phase $\phi_{r}$, and (c) reflected group delay $\tau_{r}$ as functions of frequency at different Fermi energies for TM polarized wave. Other parameters are the same as before.

refractive index supporting the excitation of SPPs is about $n_{\mathrm{SP}} \approx 3.106$ when the incident frequency equals $5 \mathrm{THz}$. Hence, it can be inferred that the excitation of SPPs is located at $\theta_{\mathrm{SP}}=\sin ^{-1}\left(n_{\mathrm{SP}} / n_{c}\right) \approx 54^{\circ}$, which is consistent with the position of the dip in Figure 2(a).

3.2. Effects of Fermi Energy on Group Delay. The excitation of SPPs in Otto configuration creates conditions for the occurrence of large reflected group delay, which can steepen the reflected phase at the excitation position, thus making it possible to have large reflected group delay. The reflectance, reflected phase, and reflected group delay in the configuration all vary with frequency; the variation relationship is shown in Figure 3. For convenience, the angle of incidence is assumed as $54^{\circ}$. As shown in Figure 3(a), a remarkable dip of the reflectance is found at the excitation spectrum of SPPs due to SPPs' excitation. This dip is resulted from the interaction of the real part and the imaginary part of the reflectance. Near the frequency that excites SPPs, the real part of the reflectance approaches zero, while the imaginary part has a remarkable monotone increase, leading to a steep monotone decrease tendency of the reflectance-based reflected phase near SPPs excitation frequency as shown in Figure 3(b). According to (3), the above phenomenon will result in large negative group delay as shown in Figure 3(c). Therefore, it can be inferred that a reflected group delay above -3 ps can be realized at $5 \mathrm{THz}$ when Fermi energy satisfies $E_{F}=1 \mathrm{eV}$. Based on the electrical tunable characteristics of graphene's optical conductivity, the group delay characteristics of the configuration can be flexibly manipulated by adjusting the Fermi energy of graphene. When the Fermi energy is reduced, the dip will have a blueshift. The reflected phase will be steeper, which will further enhance the negative value of the reflected group delay. For example, when Fermi energy $E_{F}=0.96 \mathrm{eV}$, the reflected group delay can reach about $-4 \mathrm{ps}$. These electrical tunable characteristics of the reflected group delay provide a means of designing flexible controllable delay devices.

3.3. Numerical Verification. In order to prove the correctness of the reflected group delay obtained from Figure 3, numerical simulations of the pulse's reflected group delay in the whole configuration are conducted based on Fourier transform method. Gaussian pulse is set as the incident pulse, whose electric-field expression can be expressed as $E_{i}(0, t)=$ $A_{0} \exp \left(-t^{2} / 2 \tau_{0}^{2}\right) \exp \left(-i \omega_{0} t\right)[30]$, where $\tau_{0}$ and $\omega_{0}$ represent the temporal width and center frequency of the Gaussian pulse, respectively. The corresponding Fourier spectrum of the Gaussian pulse can then be written as $E_{i}(0, \omega)=$ $\left(\tau_{0} A_{0} / 2 \sqrt{\pi}\right) \exp \left[-\tau_{0}^{2}\left(\omega-\omega_{0}\right)^{2} / 2\right]$. Large temporal width is selected to reduce the pulse distortion in the frequency domain during the reflection in the configuration. In this paper, it is assumed that $\tau_{0}=50 \mathrm{ps}$. The value of the reflected group delay of pulse can be obtained quantitatively by calculating the variation relationship between the reflected pulse and the frequency. Figure 4 is an illustration of the numerical results of normalized group delay under different Fermi energies, corresponding to Figure 3. It can be seen that the reflected group delay time obtained by simulation has a close relationship with Fermi energy. The group delay time of -3 ps (Figure 4(a)), -3.24 ps (Figure 4(b)), -3.52 ps (Figure $4(\mathrm{c})$ ), and -3.85 ps (Figure $4(\mathrm{~d})$ ) can be obtained in 


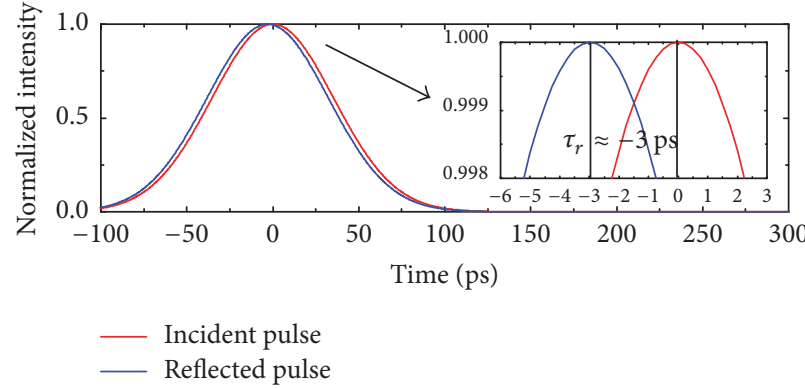

(a)

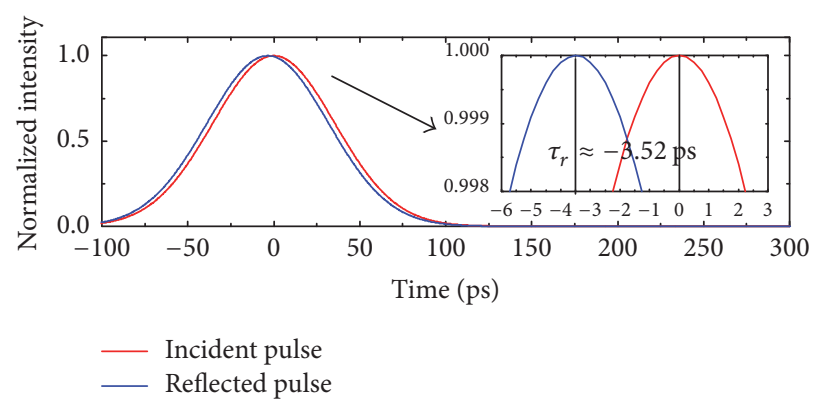

(c)

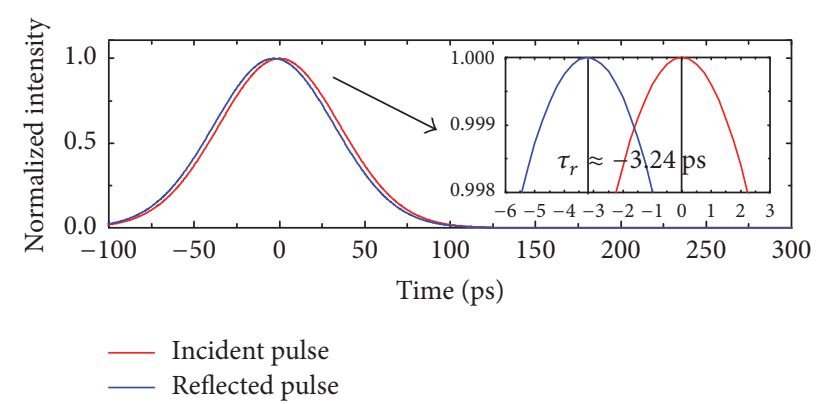

(b)

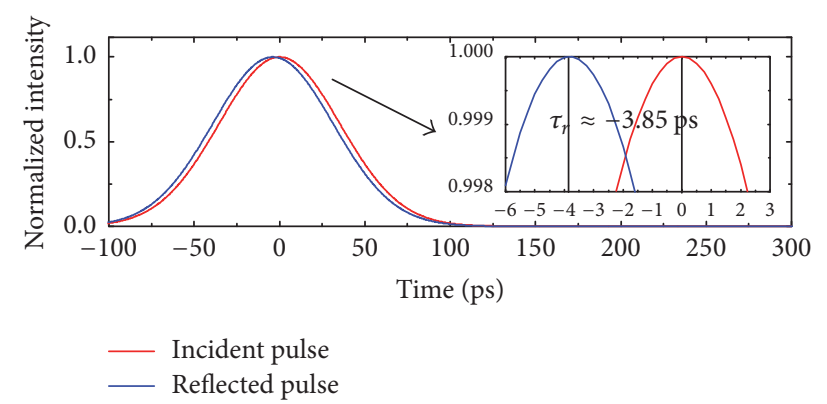

(d)

FIGURE 4: Normalized intensity of incident pulse (red line) and corresponding reflected pulse (blue line) for $p$-polarized wave with (a) $E_{F}=$ $1.02 \mathrm{eV}$, (b) $1 \mathrm{eV}$, (c) $0.98 \mathrm{eV}$, and (d) $0.96 \mathrm{eV}$. Here, (a) $\omega_{0}=2 \pi \times 5.16 \mathrm{THz}$, (b) $2 \pi \times 5 \mathrm{THz}$, (c) $2 \pi \times 4.83 \mathrm{THz}$, and (d) $2 \pi \times 4.66 \mathrm{THz}$, respectively.

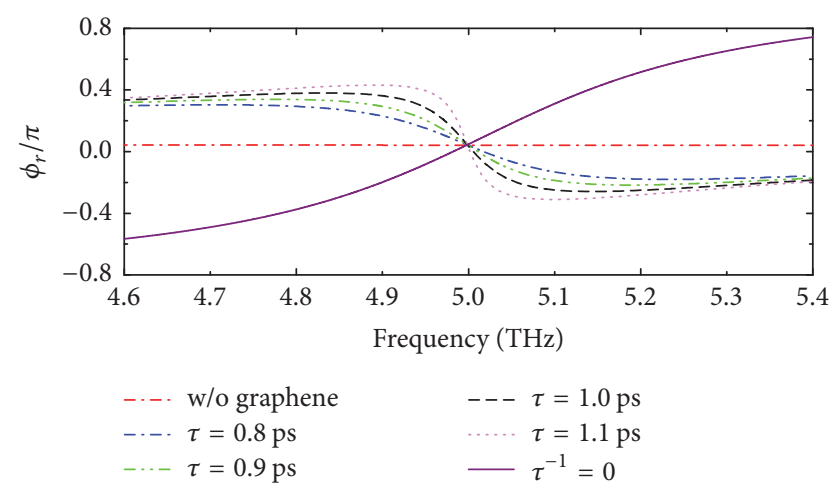

(a)

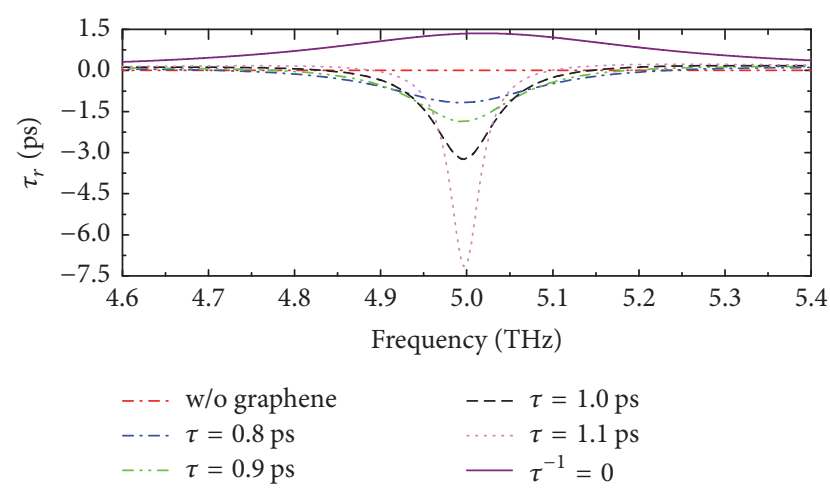

(b)

FIGURE 5: The dependence of the reflected phase $\phi_{r}$ (a) and reflected group delay $\tau_{r}$ (b) on the frequency at different relaxation times $\tau$ of the graphene. Other parameters are the same as before.

case of $E_{F}=1.02 \mathrm{eV}, E_{F}=1.0 \mathrm{eV}, E_{F}=0.98 \mathrm{eV}$, and $E_{F}=$ $0.96 \mathrm{eV}$. The group delay time is consistent with the results in Figure 3, which further proves the correctness of the delay time of Figure 3.

3.4. Effects of Other Parameters on Group Delay. According to (1), the relaxation time of graphene also has a critical impact on the optical conductivity characteristics of graphene because the actual value of the optical conductivity of graphene is strongly dependent on $\tau$. Besides, the change of optical conductivity will affect the reflection coefficient through the transfer matrix, thus affecting the reflected phase and reflected group delay. Hence, the reflected phase and reflected group delay are also sensitive to the relaxation time $\tau$. These characteristics provide a new means of regulating the group delay characteristics of reflected pulse. The change rules of the reflected phase and reflected group delay under different relaxation time are presented in Figure 5. Different from the impacts of Fermi energy on reflected phase and reflected group delay, the effect of relaxation time on reflected group delay mainly focuses on the length of the delay time rather than the frequency. Furthermore, it is found that the change of graphene's relaxation time can realize the reversal of the monotony of reflected phase, thus achieving the conversion of the positive and negative values of reflected group delay. As shown in Figure 5(a), when graphene is missing from the configuration, the reflected phase near the resonance frequency is a small value which would hardly 


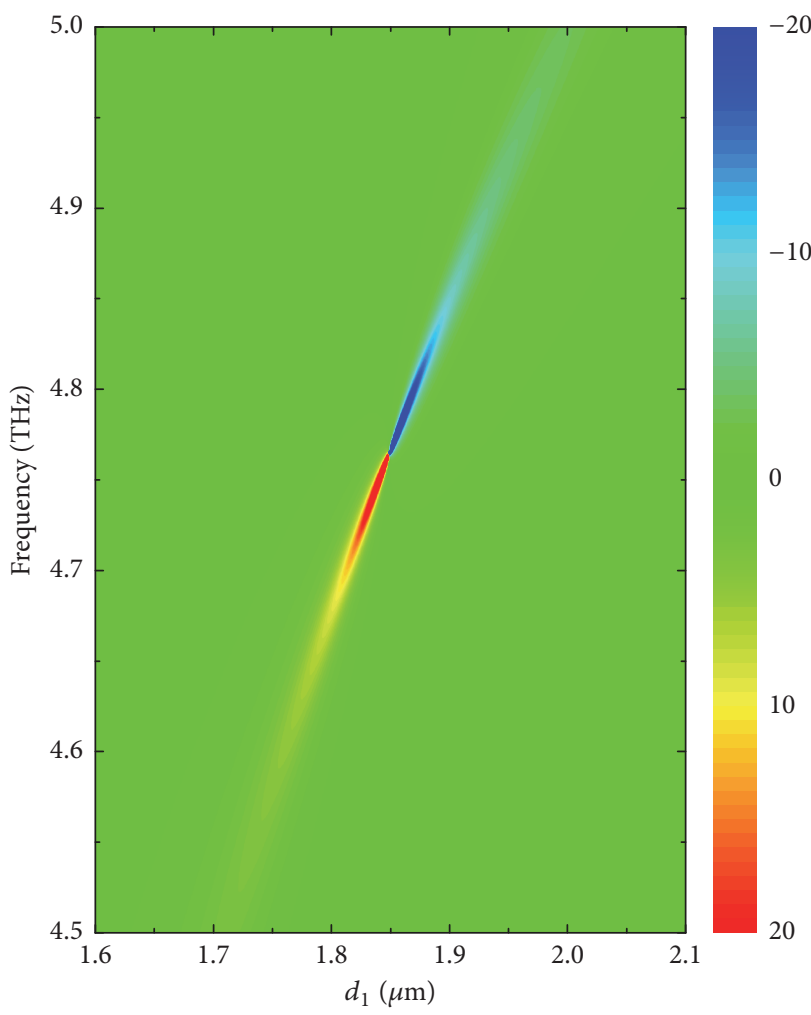

(a)

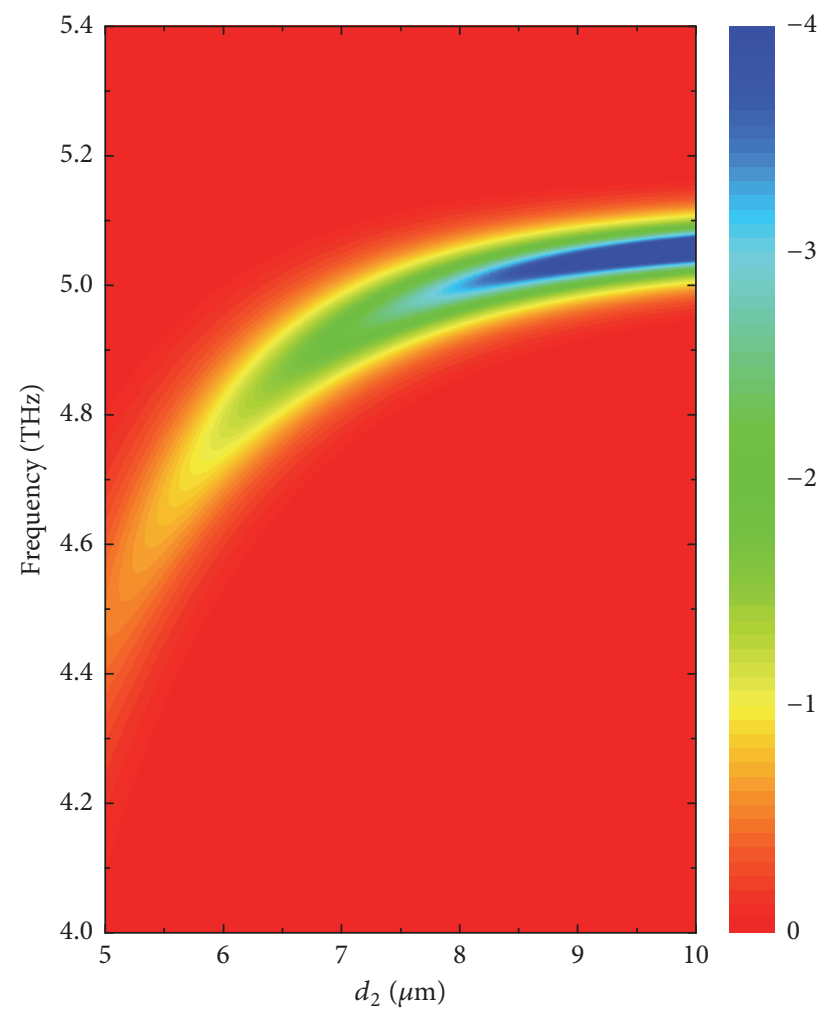

(b)

Figure 6: (a) Reflected group delay $\tau_{r}$ as a function of frequency and $d_{1}$ for the structure with the monolayer graphene. (b) Reflected group delay $\tau_{r}$ as a function of frequency and $d_{2}$ for the structure with the monolayer graphene. Other parameters are the same as before.

change. In this case, the group delay time will be very small as well. However, when graphene is included in this configuration, even small relaxation time can make the reflected phase approximate to zero and possess monotone decreasing characteristic near resonance frequency. This property indicates that the configuration has large negative delay time near the resonance point, and this negative delay time would increase remarkably near the resonant frequency with the increase of relaxation time. Particularly, the reflected phase will turn to monotone increasing near resonance frequency due to the pure imaginary characteristic of graphene's conductivity, thus providing conditions for positive reflected group delay. This tunable characteristic is an effective means of converting the delay time symbols. However, it should be noted that it will be very hard to change the relaxation time of the configuration after the preparation of actual group delay devices. Hence, in fixed configuration, the relaxation time has no tuning effect on the group delay characteristics of the whole configuration.

In the following part, the impact of the parameters of Otto configuration on the overall reflected group delay will be discussed. The conclusions can be used for important references in the design of reasonable group delay devices. Figure 6 is the contour plot of reflected group delay of dielectric slabs 1 and 2 thickness under different frequencies. By comparison, it is found that different thicknesses of the dielectric slab have different influences on the reflected group delay, while presenting varied characteristics. Dielectric slab 1 has a direct impact on the excitation of SPPs; hence the change of its thickness is very sensitive to the characteristics of the reflected group delay. As shown in Figure 6(a), when the thickness is large (e.g., larger than $2 \mu \mathrm{m}$ ), the condition of exciting SPPs cannot be satisfied and the group delay will approximate to zero. In contrast, the reflected group delay will increase tremendously by reducing the thickness, and it may reach an incredible -20 ps at about $1.85 \mu \mathrm{m}$. Meanwhile, large negative delay will accompany narrow group delay bandwidth. However, a further reduction of thickness may lead to the skipping of the delay time symbol, thus achieving large positive reflected group delay. Different from dielectric slab 1, the change of the thickness of dielectric slab 2 has a relatively simple impact on the reflected group delay. With the thickness of dielectric slab 1 unchanged, a proper increase of $d_{2}$ can significantly enhance the negative value of the reflected group delay, with the resonant frequency moving in the high-frequency direction. But when the thickness of $d_{2}$ reaches a certain level, the reflected group delay value and resonant frequency will tend to be stable. Hence, it can be concluded that a proper $d_{2}$ thickness is a necessary condition to obtain an enhanced reflected delay, which should be taken into consideration in the design of related delay devices.

\section{Conclusions}

In this paper, the enhanced group delay of pulse reflection is investigated and the surface plasmon is excited in a modified Otto configuration. It is found that the minimum value of 
reflected delay time can reach about -4 ps near the resonant angle. Simulation results reveal that the drastic change of phase obtained by the excitation of surface plasmon with graphene is the main factor in the enhancement and tuning of the reflected group delay properties. The enhanced group delay depends on the conductivity characteristic of graphene at the center frequency. The findings reveal that the group delay depends on the distance between the coupling prism and the graphene, and the thickness of substrate, above which the huge delay is observed. These findings could provide an effective method for enhancing the group delay of optical pulse and offer a feasible pathway for manipulating the delay properties. It is believed that the tunable reflected group delay at terahertz bands could contribute to the application of optical delay devices.

\section{Conflicts of Interest}

The authors declare that they have no conflicts of interest.

\section{Acknowledgments}

This work is partially supported by the Hunan Science and Technology Plan Project: Research on Key Technologies of photovoltaic inverter system (2015GK3035), the National Natural Science Foundation of China (Grant nos. 11647135 and 11704119), the Scientific Research Fund of Hunan Provincial Education Department (Grant no. 17C0945), and University Student Innovation Program of Hunan Normal University (Grant no. 2017090).

\section{References}

[1] M. S. Bigelow, N. N. Lepeshkin, and R. W. Boyd, "Superluminal and slow light propagation in a room-temperature solid," Science, vol. 301, no. 5630, pp. 200-202, 2003.

[2] R. W. Boyd, D. J. Gauthier, and A. L. Gaeta, "Applications of slow-light in telecommunications," Optics Photonics News, vol. 17, no. 4, pp. 18-23, 2006.

[3] Y. Okawachi, M. S. Bigelow, J. E. Sharping et al., "Tunable alloptical delays via brillouin slow light in an optical fiber," Physical Review Letters, vol. 94, no. 15, 2005.

[4] H. Mirzaei and G. V. Eleftheriades, "Arbitrary-angle squint-free beamforming in series-fed antenna arrays using non-Foster elements synthesized by negative-group-delay networks," Institute of Electrical and Electronics Engineers Transactions on Antennas and Propagation, vol. 63, no. 5, pp. 1997-2010, 2015.

[5] T. F. Krauss, "Why do we need slow light?" Nature Photonics, vol. 2, no. 8, pp. 448-450, 2008.

[6] J. Gu, R. Singh, X. Liu et al., "Active control of electromagnetically induced transparency analogue in terahertz metamaterials," Nature Communications, vol. 3, article 1151, 2012.

[7] L.-G. Wang and S.-Y. Zhu, "Superluminal pulse reflection from a weakly absorbing dielectric slab," Optics Letters, vol. 31, no. 14, pp. 2223-2225, 2006.

[8] L. Jiang, X. Dai, Y. Xiang, and S. Wen, "Tunable group delay of the optical pulse reflection from fabry-perot cavity with the insertion of graphene sheets," IEEE Photonics Journal, vol. 6, no. 6, 2014.
[9] S. Dutta Gupta, R. Arun, and G. S. Agarwal, "Subluminal to superluminal propagation in a left-handed medium," Physical Review B, vol. 69, no. 11, 2004.

[10] S. Manipatruni, P. Dong, Q. Xu, and M. Lipson, “Tunable superluminal propagation on a silicon microchip," Optics Letters, vol. 33, no. 24, pp. 2928-2930, 2008.

[11] H. Choi, Y. Jeong, C. D. Kim, and J. S. Kenney, "Efficiency enhancement of feedforward amplifiers by employing a negative group-delay circuit," IEEE Transactions on Microwave Theory and Techniques, vol. 58, no. 5, pp. 1116-1125, 2010.

[12] H.-Y. Yao, N. C. Chen, T.-H. Chang, and H. G. Winful, "Tunable Negative Group Delay in a Birefringent Fabry-PérotLike Cavity with High Fractional Advancement Induced by Cross-Interference Effect," IEEE Transactions on Microwave Theory and Techniques, vol. PP, no. 99, 2016.

[13] F. Bonaccorso, Z. Sun, T. Hasan, and A. C. Ferrari, "Graphene photonics and optoelectronics," Nature Photonics, vol. 4, no. 9, pp. 611-622, 2010.

[14] L. A. Falkovsky and A. A. Varlamov, "Space-time dispersion of graphene conductivity," The European Physical Journal B, vol. 56, no. 4, pp. 281-284, 2007.

[15] Z. Q. Li, E. A. Henriksen, Z. Jiang et al., "Dirac charge dynamics in graphene by infrared spectroscopy," Nature Physics, vol. 4, no. 7, pp. 532-535, 2008.

[16] Q. Bao, K. P. Loh, G. Eda, and M. Chhowalla, "Graphene photonics, plasmonics, and broadband optoelectronic devices," ACS Nano, vol. 6, pp. 3677-3694, 2012.

[17] A. H. Castro Neto, F. Guinea, N. M. R. Peres, K. S. Novoselov, and A. K. Geim, "The electronic properties of graphene," Reviews of Modern Physics, vol. 81, no. 1, pp. 109-162, 2009.

[18] N. M. R. Peres, Y. V. Bludov, J. E. Santos, A.-P. Jauho, and M. I. Vasilevskiy, "Optical bistability of graphene in the terahertz range," Physical Review B - Condensed Matter and Materials Physics, vol. 90, no. 12, Article ID 125425, 2014.

[19] S. A. Mikhailov and K. Ziegler, "New electromagnetic mode in graphene," Physical Review Letters, vol. 99, no. 1, Article ID 016803, 2007.

[20] F. H. L. Koppens, D. E. Chang, and F. J. García de Abajo, "Graphene plasmonics: a platform for strong light-matter interactions," Nano Letters, vol. 11, no. 8, pp. 3370-3377, 2011.

[21] Y. V. Bludov, M. I. Vasilevskiy, and N. M. R. Peres, "Tunable graphene-based polarizer," Journal of Applied Physics, vol. 112, no. 8, Article ID 084320, 2012.

[22] X. Dai, L. Jiang, and Y. Xiang, "Tunable THz angular/frequency filters in the modified kretschmann-raether configuration with the insertion of single layer graphene," IEEE Photonics Journal, vol. 7, no. 2, 2015.

[23] H. Wang, J. Wu, J. Guo, L. Jiang, Y. Xiang, and S. Wen, "Lowthreshold optical bistability with multilayer graphene-covering Otto configuration," Journal of Physics D: Applied Physics, vol. 49, no. 25, Article ID 255306, 2016.

[24] Y. Luo, V. K. Guda, E. B. Hassan, P. H. Steele, B. Mitchell, and F. Yu, "Hydrodeoxygenation of oxidized distilled bio-oil for the production of gasoline fuel type," Energy Conversion and Management, vol. 112, pp. 319-327, 2016.

[25] B. Ding, Y. Cheng, J. Wu et al., "A unique multifunctional cluster-based nano-porous Terbium organic material: Realtime detection of benzaldehyde, visually luminescent sensor for nitrite and selective high capacity capture of Congo Red," Dyes and Pigments, vol. 146, pp. 455-466, 2017. 
[26] Q. Xu, H. Xu, J. Chen, Y. Lv, C. Dong, and T. S. Sreeprasad, "Graphene and graphene oxide: Advanced membranes for gas separation and water purification," Inorganic Chemistry Frontiers, vol. 2, no. 5, pp. 417-424, 2015.

[27] L. Jiang, J. Guo, Q. Wang, X. Dai, and Y. Xiang, "Perfect Terahertz Absorption with Graphene Surface Plasmons in the Modified Otto Configuration," Plasmonics, pp. 1-7, 2016.

[28] Y. Xiang, X. Dai, J. Guo, S. Wen, and D. Tang, "Tunable optical bistability at the graphene-covered nonlinear interface," Applied Physics Letters, vol. 104, no. 5, Article ID 051108, 2014.

[29] T. Zhan, X. Shi, Y. Dai, X. Liu, and J. Zi, “Transfer matrix method for optics in graphene layers," Journal of Physics Condensed Matter, vol. 25, no. 21, Article ID 215301, 2013.

[30] L.-G. Wang, H. Chen, and S.-Y. Zhu, "Superluminal pulse reflection and transmission in a slab system doped with dispersive materials," Physical Review E - Statistical, Nonlinear, and Soft Matter Physics, vol. 70, no. 6, Article ID 066602, p. 066602/6, 2004. 

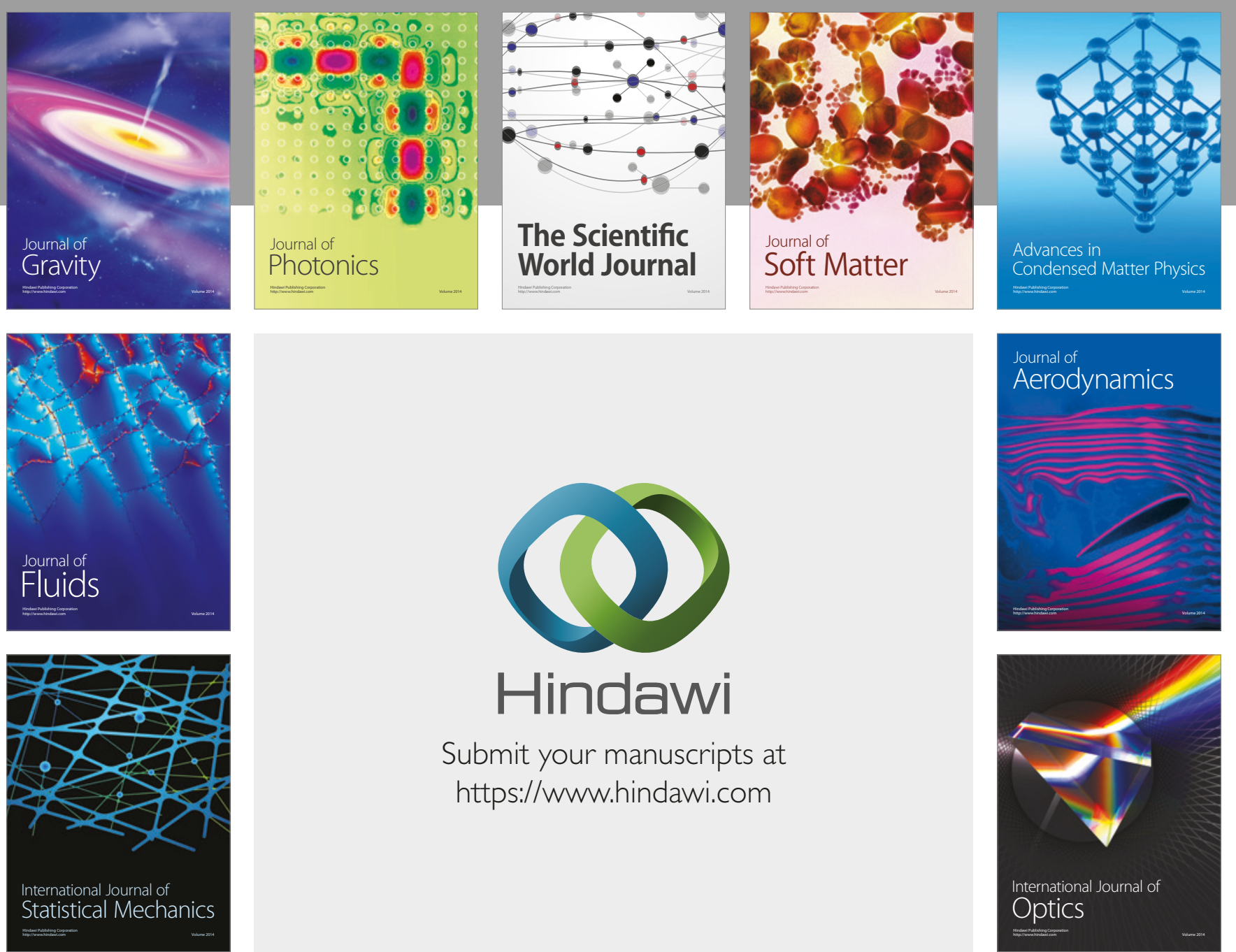

Submit your manuscripts at

https://www.hindawi.com
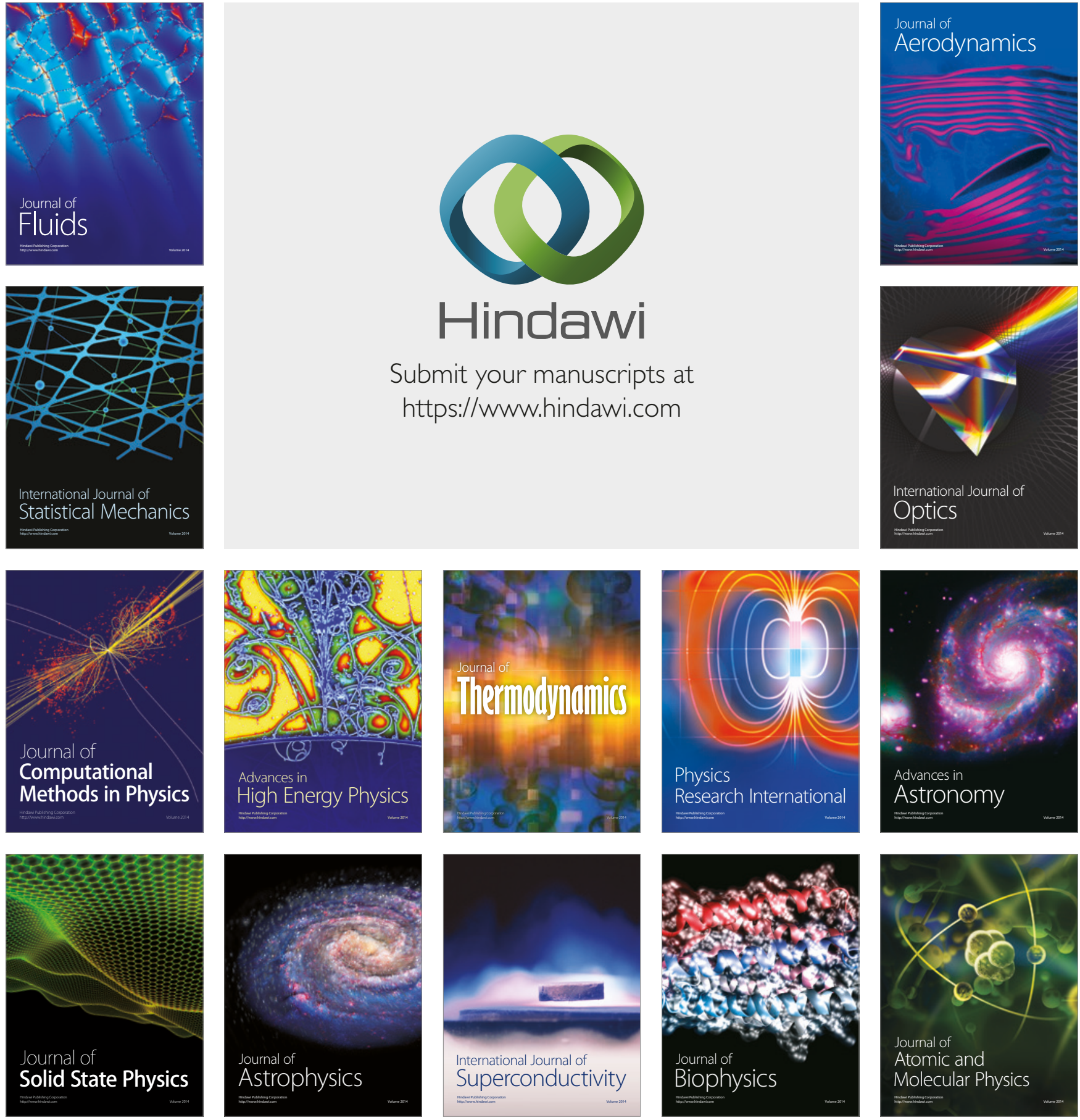